\title{
Histochemical, ultrastructural and structural changes in primary cardiomyopathy and in cobalt cardiomyopathy
}

\author{
ECKHARDT G. J. Olsen
}

THE paper on 'The pathology of primary cardiomyopathy' (page 732) concentrated on the criteria useful for diagnosis. This paper deals with the histochemical and ultrastructural changes (Van Noorden, Olsen \& Pearse, 1971)

\section{Congestive cardiomyopathy (systolic pump failure)}

Glycogen is patchily increased in the myocardial fibres surrounding the nucleus and may also be found between myocardial fibrils. As a marker of mitochondrial activity, succinic dehydrogenase and NADH diaphorase are used which show a similar distribution to that of glycogen, i.e. increased activity can be seen in the perinuclear region and between fibrils. Acid phosphatase and non-specific esterases used as markers for lysosomal activity show also increased activity. The distribution is patchy throughout biopsy material. The histochemical changes reflect hypertrophy of the myocardium but we have been unable to find a specific enzyme alteration pathognomonic for congestive cardiomyopathy.

Ultrastructural changes consist of an increase in mitochondria. In some instances mild mitochondrial swelling is evident with some degenerative changes in cristae and occasionally mitochondrial 'ghosts' are seen. An increase in lipofuscin granules and lipofuscin precursor granules has also been found. The contractile elements are preserved and long runs of fibrils are seen.

Hypertrophic cardiomyopathy with or without obstruction (diastolic compliance failure)

The most striking feature on histochemical examination is the glycogen content. There is considerable increase, particularly in the perinuclear area. Usually, with moderate amounts of increase in glycogen, a granular appearance is observed, but in cases of hypertrophic cardiomyopathy with obstruction, the granularity is lost and large areas of 'smudges' are seen, the so-called pooling of glycogen. Figure 1 compares the glycogen content of the heart with that of ordinary hypertrophy.

Histochemical analysis of many other systems, such as succinic dehydrogenase, acid phosphatase and non-specific esterases reveals quantitative changes consistent with the extreme hypertrophy seen in some myocardial fibres.
Ultrastructural changes may be striking but are unfortunately in no way pathognomonic for the condition. The uniform parallel arrangement of the contractile elements is lost and fibrils run in all directions (Fig. 2). Similar changes affecting fibres are seen at light microscopic level.

Dystrophic changes of contractile elements are often observed but a dystrophic fibril may lie adjacent to a normal fibril. This patchy distribution makes interpretation of the appearances, particularly on needle biopsy, extremely difficult.

Extreme apparent increases in mitochondria, the so-called mitochondriosis, are frequently seen. Each of these is within the lower limit of normal dimensions. Cross-over of myofilaments has been demonstrated (Ferrans, 1971) and this network of crossing contractile elements may explain the clinica manifestations. These changes can however also be seen in ordinary hypertrophy.

The histochemical changes allow one to understand some of the processes which occur within the myocardial cell and electron-microscopic investigations show the morphological appearances at very high magnification. These changes are, however, descriptive, and future work should concentrate on biochemical analysis which might throw light on this puzzling condition. It has previously been emphasized that the asymmetric hypertrophy of the interventricular septum is usually evident, even if clinically the obstructive element had disappeared.

There is, however, a very small number of patients who do not fit into the category of the hypertrophic obstructive group, nor do they fit into the congestive group. These patients are extremely difficult to classify and usually have some clinical features of obstruction with dominant congestive failure (Karatzas, Hamill \& Sleight, 1968).

At necropsy these patients show hypertrophy and often extreme dilatation of all the chambers, together with endocardial thickening with or without superimposed thrombus. Macroscopically they resemble the congestive group of cardiomyopathies but they differ from congestive cardiomyopathy on histological examination.

Histologically this rare group show foci of abnormal cells indentical to those described in the 

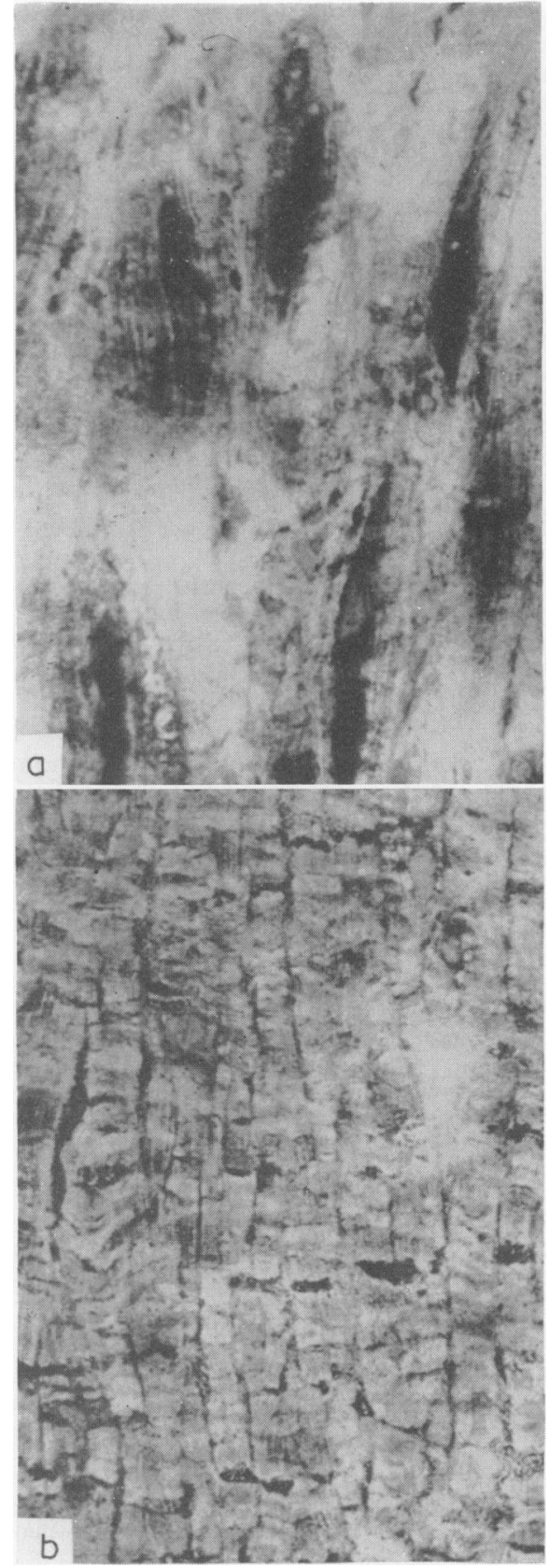

Fig. 1. Photomicrograph to compare glycogen content of myocardial hypertrophy. (a) Hypertrophic cardiomyopathy with obstruction, showing pooling of glycogen in the perinuclear area. (b) Hypertrophy due to aortic valve stenosis (periodic acid Schiff, $\times 330$ ).

hypertrophic obstructive cardiomyopathic group. These foci of abnormal fibres, which are not seen in typical congestive cardiomyopathies, are randomly

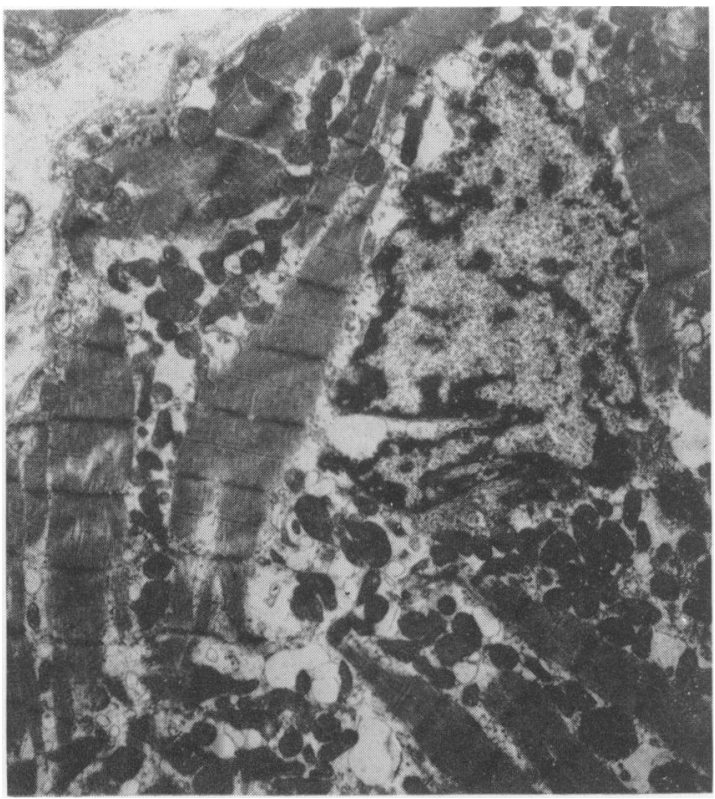

FIG. 2. Electron-micrograph from a biopsy of a patient with hypertrophic cardiomyopathy with obstruction. The myocardial fibrils run in all directions. The mitochondria are only moderately increased in this field. The nuclear membrane shows crenation (lead citrate and uranyl acid, $\times 8750$ ).

distributed, usually in the left ventricular myocardium, including the lateral wall (Fig. 3). This group suggests that a spectrum of hypertrophic obstructive cardiomyopathy may exist. On the one end of the spectrum the typical asymmetric hypertrophy (which may disappear) is seen, but in these patients, the distribution of abnormal fibres is usually in one continuous band, most commonly in the interventricular septum. At the other end of the spectrum there are these small foci of randomly distributed abnormal cells, and it may be that the different distribution may explain the different clinical manifestations (Olsen, 1971).

\section{Alcoholic cardiomyopathy in beer drinkers-the cobalt story}

In 1967 a report from Quebec described a cardiomyopathy affecting patients who had taken up to 20 pints of beer a day (Morin et al., 1967). The causative agent was eventually traced to cobalt which had been added to the beer in order to make it more frothy. The Quebec group of workers have been able to examine twenty-five hearts at necropsy; the hearts weighed $350-690 \mathrm{~g}$. There was some dilatation of the ventricles, the myocardium showed moderate hypertrophy and occasionally ventricular mural thrombi were present. Histological examination 


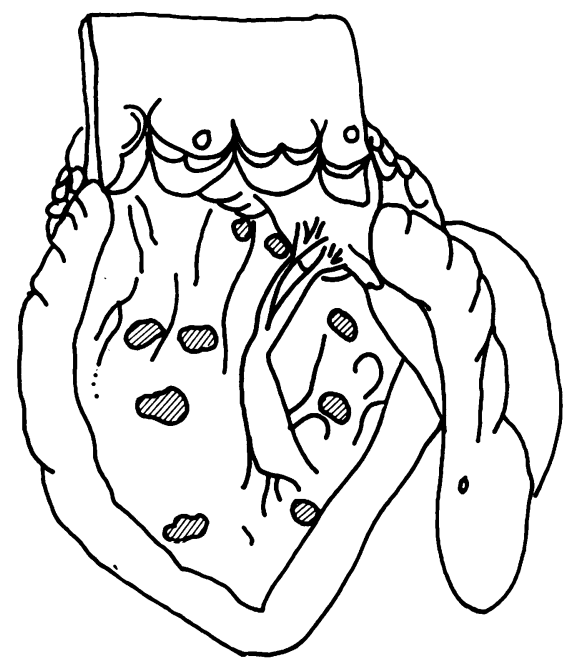

Fig. 3. Diagrammatic representation of the left ventricle showing foci of abnormal fibres identical to those described in the 'classical' form of hypertrophic cardiomyopathy with obstruction. Compare with Fig. 4, page 734 .

showed characteristic changes (Bonenfant, Miller \& Roy, 1967). Variation in the staining properties of hypertrophic myocardial fibres was evident; the size of the muscle fibres varied and there was a decrease in myofibrils. Vacuolation within the myocardial fibre, sometimes multiple and small and sometime large, was invariably present (Fig. 4). The vacuoles were due, at least in part, to glycogen accumulation. Intracellular oedema was observed in myocardial fibres separating individual fibrils from one another. Interstitial oedema is also seen. Focal fibrosis of varying severity was present and a striking feature was the virtual absence of inflammatory change. Hyaline change of myocardial fibres was observed. The nuclei were occasionally bizarre in shape but the marked changes seen in cases with hypertrophic cardiomyopathy with obstruction, and the extreme hypertrophy of myocardial fibres were not seen.

Experimental support for the hypothesis that cobalt could be the underlying cause for the cardiomyopathy was obtained by feeding rats on a proteindeficient diet and administering cobalt (Rona \& Chappel, 1971). The features described histologically by the Quebec workers were simulated in these studies. At electron-microscopic level, swelling of mitochondria and occasional distortion of cristae and the outer membrane was noted. In addition to

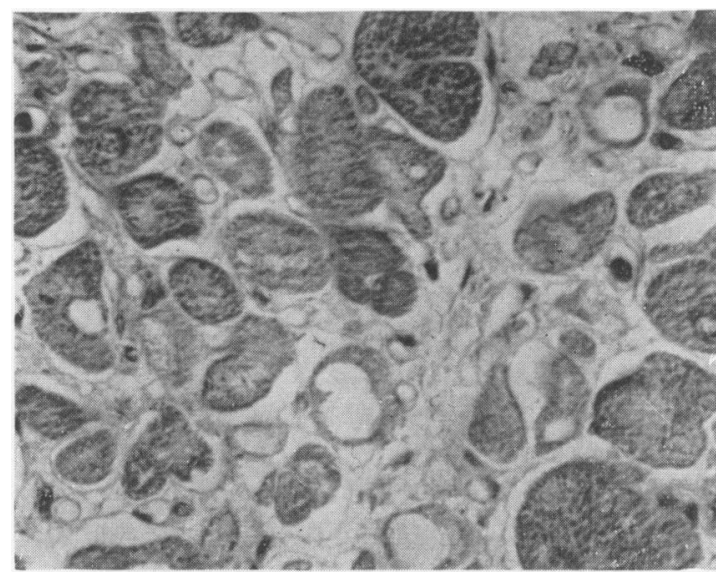

FIG. 4. Photomicrograph of cobalt cardiomyopathy. Note the intracellular oedema separating individual myocardial fibrils and the variously-sized vacuolation of myocardial fibres $(\mathrm{H} \& \mathrm{E}, \times \mathbf{2 1 0})$.

the changes in mitochondria, a pathognomonic feature for cobalt toxicity was found, consisting of dense, osmophilic, intramitochondrial particles $0 \cdot 3 \cap \frac{\Phi}{-}$ $0.4 \mu \mathrm{m}$ in diameter. It has been suggested that these particles represent cobalt-protein complexes and no calcium.

\section{Acknowledgments}

This work has been carried out in close collaboration with Miss Susan Van Noorden and Professor A. G. E. Pearse. I am grateful to Dr G. Miller for the material on cobalt cardiomyopathy and to Professor G. Rona for electronmicroscopic preparations.

\section{References}

Bonenfant, J.-L., Miller, G. \& Roy, P.E. (1967) Quebec beer-drinkers' cardiomyopathy. Pathological studies. Canadian Medical Association Journal, 97, 910.

Ferrans, V.J. (1971) Personal communication.

Karatzas, N.B., Hamill, J. \& Sleight, P. (1968) Hypertrophic cardiomyopathy. British Heart Journal, 30, 826

Morin, Y.L., Foley, A.R., Martineau, G. \& Roussel, J. (1967) Quebec beer-drinkers' cardiomyopathy. Forty-eight cases. Canadian Medical Association Journal 97, 881.

Noorden, S. VAN, Olsen, E.G.J. \& Pearse, A.G.E. (1971) Hypertrophic obstructive cardiomyopathy, a histological, histochemical and ultrastructural study of biopsy material. Cardiovascular Research 5, 118.

OlSEN, E.G.J. (1971) Morbid anatomy and histology in hypertrophic obstructive cardiomyopathy. In: Hypertrophic Obstructive Cardiomyopathy, Ciba Foundation Study Group No. 37, p. 183. Churchill, London.

Rona, G. \& Chappel, C.I. (1971) Personal communication. 